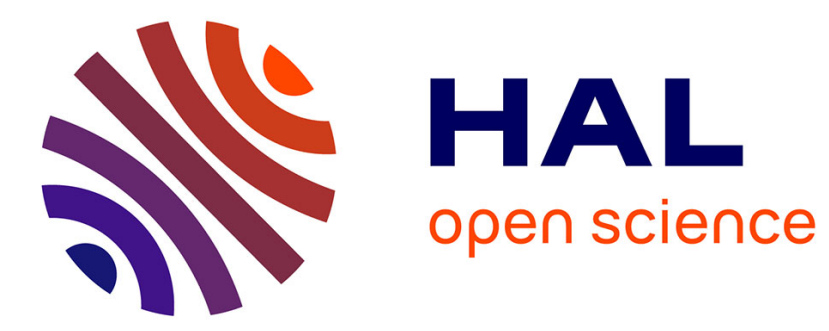

\title{
Les bijoux d'or gallo-romains de l'Houmeau (Charente-Maritime)
}

Jean Flouret, Gérard Nicolini, Catherine Metzger

\section{To cite this version:}

Jean Flouret, Gérard Nicolini, Catherine Metzger. Les bijoux d'or gallo-romains de l'Houmeau (Charente-Maritime). Gallia - Fouilles et monuments archéologiques en France métropolitaine, 1981, 39 (1), pp.85-101. 10.3406/galia.1981.1822 . hal-01940655

\section{HAL Id: hal-01940655 \\ https://hal.science/hal-01940655}

Submitted on 27 Feb 2020

HAL is a multi-disciplinary open access archive for the deposit and dissemination of scientific research documents, whether they are published or not. The documents may come from teaching and research institutions in France or abroad, or from public or private research centers.
L'archive ouverte pluridisciplinaire HAL, est destinée au dépôt et à la diffusion de documents scientifiques de niveau recherche, publiés ou non, émanant des établissements d'enseignement et de recherche français ou étrangers, des laboratoires publics ou privés.

\section{(ㅇ)(1) $\$$}

Distributed under a Creative Commons Attribution - NonCommercial - NoDerivatives $\mid 4.0$ 


\title{
LES BIJOUX D'OR GALLO-ROMAINS DE L'HOUMEAU (Charente-Maritime)
}

\author{
par Jean FLOURET, Gérard NICOLINI et Catherine METZGER
}

\section{LA DÉcouverte HT SON CONTEXTE.}

En face de Saintes et de la Saintonge au riche passé gallo-romain, la Rochelle et l'Aunis font figure de parent paure. Ln enfant du pays, le professeur rochelais Léopold Jelayant, n’allait-il pas jusqu'à affirmer, en 1870 : "On croit, en général, que les Romains se sont arrêtés dans notre pays au sud de la charente. Il faut faire une grande violence au texte de Strabon pour voir dans Charron, Marans et Chaillé, des îles du Lac des Corbeaux; mais il est bien probable qu'en effet les lieux où est aujourd'hui la Rochelle étaient alors inhabitables »'. Pourtant, dès cette époque, les découvertes étaient nombreuses dans l'Aunis et montraient avec certitude que les Romains avaient bien franchi la Charente et occupé le pays d'Aunis. Le premier à voir la vérité, et cela vingt ans avant Delayant, fut le naturaliste rochefortais René-Primevère Lesson, à qui il faut rendre hommage. I)ans ses Fasles historiques, au milieu d'une énumération déjà impressionnante de vestiges, il écrit : "J'ai trouvé tant de traces de l'occupation romaine dans l'Aunis, qu'il n'est plus possible d'admettre avec tous les auteurs qui, sans exception, ont érit sur notre province, que l'Aunis ait été inconnu aux conquérants des Gaules, possesseurs de la Saintonge $»^{2}$.

Cette occupation est amplement confirmée par les découvertes de ces dix dernières années (fig. 1), dues surtout à un regain d'intérêt pour l'histoire et l'archéologie, à une exploration systématique des terres lors des grands travaux de voirie et a l'activité des membres de la Société d'Archéologie et d'Histoire de l'Aunis, fondée en 1970. A la Rochelle même, dans le faubourg de Saint-Eloi, une villa agraire édifiée dans la seconde moitié du ${ }_{1}$ er siècle et habitée jusqu'à la fin du in ${ }^{\mathrm{e}}$ siècle, a été fouillée récemment ${ }^{3}$, tandis que sur la pointe des Minimes, un site s'étendant sur plus d'un hectare est en cours de sauvetage.

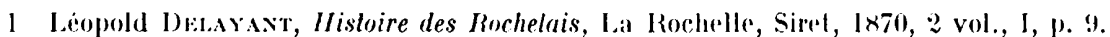

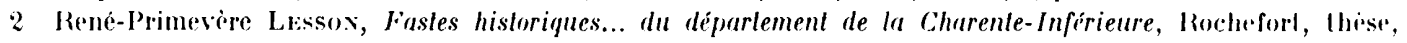
IX.12 et Proust-Branday, 18.45, 2 vol., I, p. 53.

3 Jran Frovavt, Vestiges gallo-romains a I.a Rochelle, dans Revue de la Sainlonge el de l'. Iunis, IV, I978, p. 6I. 


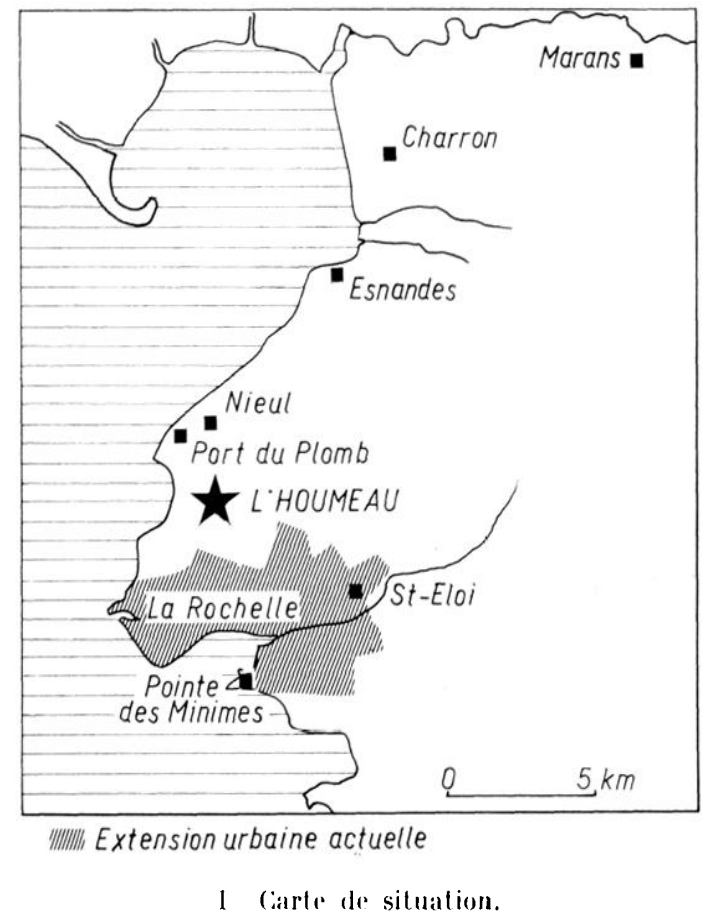

Mais c'est surtout au nord de la ville, tout le long de la côte, que l'occupation semble avoir été la plus dense ${ }^{4}$. En particulier, à Marans, de nombreuses poteries sigillées et communes, de la verrerie ont été mises au jour à la fin du siècle dernier. A Charron, sous des sépultures médiévales, une occupation continue est attestée depuis la période de llallstatts. Sur la falaise qui domine le port d'Esnandes, on distingue des murs sur une grande surface et pourtant, beaucoup de vestiges ont certainement été engloutis au cours des siècles par la mer qui a considérablement rongé en ce lieu la falaise depuis deux mille ans. A Nieul-sur-Mer, un aqueduc souterrain amenant l'eau de la fontaine de Grimaud au port du Plomb a été exploré sur plusieurs centaines de mètres ${ }^{6}$, alors que sur les hauteurs qui dominent à $18 \mathrm{~m}$ le port, à l'est, un site comprenant des substructions, un puits, deux citernes et un bassin, est en cours de fouille?

A $2 \mathrm{~km}$ an sud de . Yieul et $5 \mathrm{~km}$ an nord de La Rochelle, le village de l'lloumeau, lieu de découverte des bijoux qui font l'objet de cette élude, est installé a l'abri, au fond d'un petit estuaire marécageux, bordé par le Trépied du Plomb el par une colline qui culmine a $19 \mathrm{~m}$. Le vieux village occupe le versant nord-est de la colline. Landis que te versant sudt. aut refois voué uniquement aux cultures, se grarnil aujourd'hui de lotissements. Sur ce versanl. qui domine te marais de Pampin, on trouve à fleur de terre de nombreux tessons de céramique antique et, ces dernières années, un

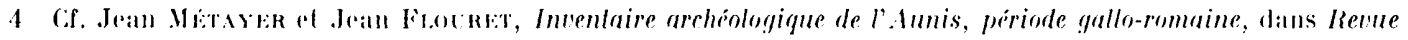
de la siainlonge el de litunis, N, 1978, p. 69.

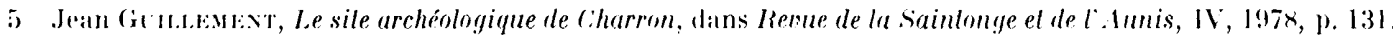

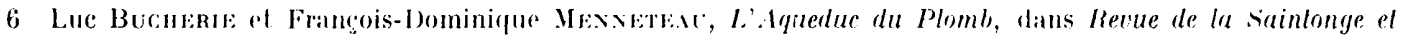
de l'Aunis, V, 1979, p. 119.

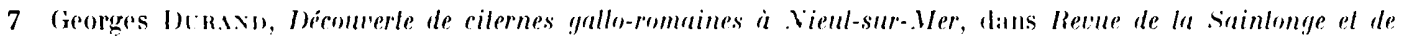
l.Aunis, II, 1976, p. 13.4. 
sesterce de Marc Aurèle et un autre de Faustine mère, postérieur à 141, ont été découverts. Enfin, au début de 1979. en creusant les fondations d'une maison, rue Jean-Bart, les pelleteuses ont sectionné des citernes visiblement de construction gallo-romaine. Cràce à l'amabilité des propriétaires, II. et . I ${ }^{\text {me }}$ Joël André, une étude rapide du fond des citernes a pu ètre menée à bien par quelques membres de la société d'Archéologie el d'Histoire de l'Aunis, en particulier IIme Lafon et M. M. Texier et Laporte, avec l'accord de la Direction régionale des Antiquités, prévenue aussitòt. Jeux citernes parallèles, ont livré une cruche en céramique commune, des tessons et deux serpettes. Une troisième citerne, primilivement carrée, puis surélevée, ağrandie et rendue rectangulaire, ne contenait que quelques tessons et fonds de vases. Enfin, dans le mème jardin, on trouva un sesterce de Gordien (Atelier de Rome, 2:38-239, R.I.C.., no 2:4), une picce de Gallien (Atelier de Rome. 2633, R.I.C., $\mathrm{n}^{0} 227$ ) et une autre de Tétricus (Imilation du type Salus??.

C'est dans ce contexte archéologique, à quelques mètres des citernes, que M. Georges Durand, secrétaire de la Société d'Archéologie et d'Histoire de l’Aunis, a mis au jour un petit trésor constitué par six bijoux. (irâce à la compréhension de II. et II me André, ces

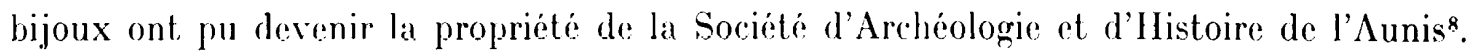

Le trésor élail enfoui à une vingtaine dre centimètres du sol, protégé par une grosse pierre. Jeux médaillons monétaires, contenant, le premier un denier de Septime Sévère, l'autre un denier de Caracalla, recouverts d'une mince feuille d'or estampée sur la pièce, étaient placés l'un au-dessus de l'autre. Les quatre autres éléments du trésor, coulant, nœud d'Héraklès et deux heptagones, étaient placés tout contre les médaillons. L'ensemble avait peut-ètre été enveloppi dans un chiffon ou un sac. Ln des médaillons avait étéécrasé. ainsi que le coulant, et le denier. au contact de la terre, avait produit de l'oxydation qui avait taché l'ensemble des bijoux. Il a donc fallu démonter et restaurer le médaillon abîmé, nettoyer tous les bijoux : e travail a été exécuté par MII. Pierre et Claude Billard, bijoutiers à la Rochelle.

J. F.

\section{II. Étene techingre.}

L'étude technique de ces pièces a été réalisée à partir d'observations à la loupe binoculaire, de macrophotographies que nous présentons ici accompagnées de leur échelle évaluée au point de netteté de l'épreuve, et enfin d'analyses pratiquées par le laboratoire des Musées de France par la méthode de la microfluorescence X (recherche de l'argent et du cuivre donnés ici en pourcentage) et celle de la spectrographie d'émission L.V. (traces de métaux et métalloïdes) ${ }^{9}$. La multiplicité des points d'analyse sur les différentes pièces nous a permis de pereroir des variations de composition appréciables, et quelquefois signilicatives. Nous étudions chacune de celles-ci en considérant l'état de conservation, la nature du métal, et la technique de fabrication.

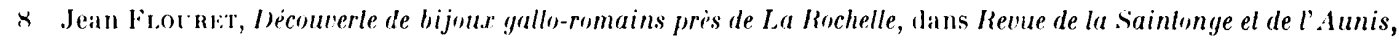
V, 1979, p. 3-7.

9 . Tous remercions bien vivement 11 me .I. Hours qui a bien voulu accepter de pratiquer ces analyses ainsi que M.I. Ch. Lahanier et L. Hurtel, qui nous ont donné d'utiles indications techniques. Les résultats que nous présentons ici sont extraits des tableaux (qu'ils nous ont aimab)ement communiqués. 
(Les principales abréviations utilisées sont : $\mathrm{Ag}$ : argent, Cu : cuivre, Sn : étain, $\mathrm{Pb}$ : plomb, Zn : zinc, lid : cadmium, Fr: fer, Ni : nickel, Vn : manganèse).

1. Médaillon monélaire (denier de Septime Sévère) (fig. 2 à 4, 18, 20,21).

Dimensions : haut. totale : $29,5 \mathrm{~mm}$; diam. : 25,5 environ; épais. (cylindre) : 5 environ; poids : 7,67 g. Atelier de Rome, 201-210, type B.M.C. no 334, Cohen no 217.

D/ SEVERVS PIVS AVG. Tête lauréc à droite.

R/ INIDVIGE NTIA AVG(i. Exergue : IN CARTH.

Élal de conservalion. Yonnaie : sur la macrophotographie (fig. 18) et à la loupe binoculaire, la surface de la feuille de placage apparait granuleuse; reliefs usés. Cylindre: usure, surtout du côté du revers, par le port; coups et fentes (fig. 20). Biseau côté du droit : rayure en haut. Biseau rôté du revers : fentes et desquamations. Collerette : usure et fentes sur le bord extérieur; nombreuses adjonctions de fragments de feuille qui sont peut-être des réparations ou des consolidations antiques, particulièrement du côté du revers (fig. 4, 18). Bélière : remise en forme et ressoudée sur la collerette après la trouvaille; trace d'outil moderne sur le revers (fig. 20 ); l'usure provoquée par le cordon de suspension est visible (fig. 21$)$.

Mélal. Monnaie : l'analyse de la feuille de placage fait apparaître une disparité de la teneur en cuivre entre le droit $(\mathrm{Cu}=4,3)$ et le revers ( $\mathrm{Cu}=7,9)$, visible à l'œil, mais peu explicable. Il est rependant intéressant de constater que deux ors différents ont été utilisés ${ }^{10}$. On fera la même constatation en ce qui concerne deux autres parties de la monture, la bélière et la colleretten :

\begin{tabular}{|c|c|c|c|c|c|c|c|c|c|}
\hline & $\lambda g$ & $\mathrm{Cu}$ & $\sin$ & $\mathrm{Pb}$ & $\mathrm{Zn}$ & Crl & lie & $\mathrm{Mi}$ & .In \\
\hline Bélière............... & 7.3 & 2.9 & d & a & $d$ & d & d & $d$ & d \\
\hline Gollerette............ & 6.8 & 4.9 & $d$ & d & $d$ & d & $d$ & nd? & nd \\
\hline
\end{tabular}

Nous revenons plus bas sur la présence du zinc et du cadmium dans ces ors probablement alliés. Il faut noter l'emploi peu judicieux d'un or aussi mou pour la bélière, dont nous avons relevé l'usure. Si l'on excepte la feuille de placage du revers. la teneur en cuivre la plus élevée est celle de la collerette, mais on ne peut assurer que ce choix d'un or plus résistant ait été délibéré. Les autres parties du bijou présentent également des disparités notables. On décèle dans le cylindre. $\mathrm{Ag}=6.4$ et $\mathrm{Cu}=4.7$ et dans le biseau du droit $\mathrm{Ag}=11, \mathrm{Cu}=3,7$. La composition de ce dernier élément, nettement plus clair est là encore injustifiable.

10 Les différences de teneur en argent $\mathrm{Ag}=6,5$ a 6 sont peu significatives à cause de la perméabilité de la feuille mince aux rayons $X$. On fait la même constalation à propos du second médaillon ve infra .

11 Dans les tableaux damalyses que nous dommons infra, l'argent el le cuivere sonl exprimés en pourcentage, les autres métaux de maniere qualitalive : $d=$ decele, nd $\ldots$ non décelé. 
Technique de fabricalion. Le placage de la monnaie pose un intéressant problème de facture. La feuille est assez épaisse, semble-t-il. Il est possible qu'elle ait été estampée à l'aide du denier même qui aurait servi de poinçon, puis ajustée sur la monnaie par un procédé s'apparentant à celui du "fourré "12. Il est beaucoup plus vraisemblable qu'elle a été façonnée dans une matrice en creux, analogue à celles des imitations monétaires ${ }^{13}$, produite par l'impression du denier dans l'argile avant cuisson. La technique est connue sous le nom de "moulage à froid ". Elle est révélée ici par la surface rugueuse de la pièce, probablement due à la matrice de terre cuite, que l'orfèvre n'a pas cru bon de brunir après l'application de la feuille sur la monnaie ${ }^{14}$.

Le cylindre est fait à l'aide d'une bande d'épaisseur très irrégulière, de 5 à $15 / 100^{\mathrm{e}}$ environ, obtenue par martelage direct sur une surface granuleuse et peu régulière (fig. 20-21) mise en forme et soudée à gauche de l'avers, les bords ont été amincis par un martelage plus prononcé, probablement responsable d'une partie des fentes que l'on observe du côté du revers. I)u côté de l'avers, ils ont été évasés et roulés vers l'extérieur. Du côté opposé ils sont au contraire grossièrement rabattus vers l'intéricur.

La monnaie est maintenue à l'intérieur du cylindre par deux biseaux faits d'une bande étroite sommairement martelée comme le prouvent des marques "en vaguelettes", des fentes longitudinales sur le revers en haut (fig. 18) et surtout une épaisseur très irrégulière variant de 10 à $25 / 100^{e}$ environ. Le découpage a été fait au ciselet sans grand soin. Les extrémités de la bande ont été amincies par martelage, superposées et soudées. Sur l'avers le biseau est fixé à l'aide de soudure en six points irrégulièrement répartis. Sur le revers il ne semble pas soudé mais seulement tenu par le débord du cylindre rabattu en une sorte de serti sommaire ${ }^{15}$. La colleretle soudée autour du cylindre est constituée d'une bande martelée d'aussi piètre façon que les autres parties du bijou. Son épaisseur varie de 5 à 30/100e environ, le bord ayant été par endroit épaissi par le découpage au ciselet, dont on perçoit les traces longitudinales sur le chant. Ie gaufrage a été obtenu à l'aide d'une pince par une légère torsion. Il a servi aussi bien au décor qu'au resserrement de la collerette autour du cylindre. La soudure, à l'alliage selon toute vraisemblance, est largement débordante et "bouillie" en différents endroits, notamment sur le revers. On ne sait si cette surchauffe est maladroite ou au contraire volontaire, dans le but d'une égalisation des couleurs de la pièce et des soudures ${ }^{16}$. Enfin il est possible que l'adjonction des pièces rapportées sur le revers ait provoqué une nouvelle fusion des soudures de la collerette.

La bélière ne se présente pas dans son montage originel (roir supra), elle est faite d'une bande d'épaisseur très irrégulière au bord (de 35) à 60/100e) martelée sur un dé à

12 Sur la technique du "fourri", ef. G. Yicor.si, La bijouterie d'or ibérique, thise dactylographiéc

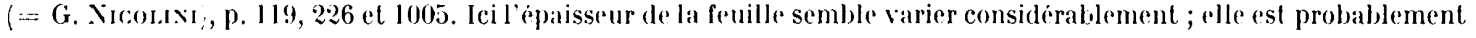
partout supérieure à $2 / 100$ e.

13 G. Brnrrss, Römische Falschmünzformen, Aclas y Vemorias de la sociedal Española de Antropologia, Elmografia y Prehistoria 21-1946, p. 89-100, pl. IV.

14 Sur le moulage à froid en général, G. Nicomsi, p. 68,111 of 1005.

15 Ibid., p. 229 el 250. Il est possible que ce serti ait été modifié légrerement lors du remontage du bijou.

16 La surchauffe a quelquefois élé pratiquée pour éliminer le cuivere dans l'alliage des soudures, qui pourraient paraitre plus foncées que la piece sans cela. Voir ibid., p. 208 et R. A. Hogiss, op. cit., p. 36, H. Maryox, Technical studies in the field of tine Arts, 5-1936, p. 91. 


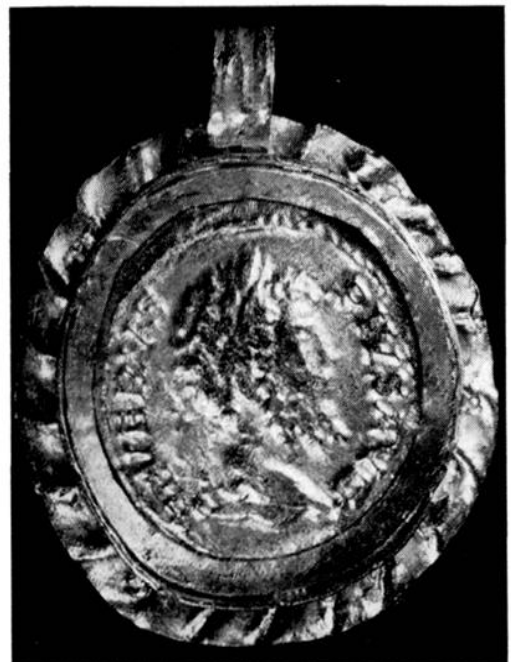

2 Vidaillon monetaire $n^{\prime \prime} 1$. Avers. (Esh. : :1).

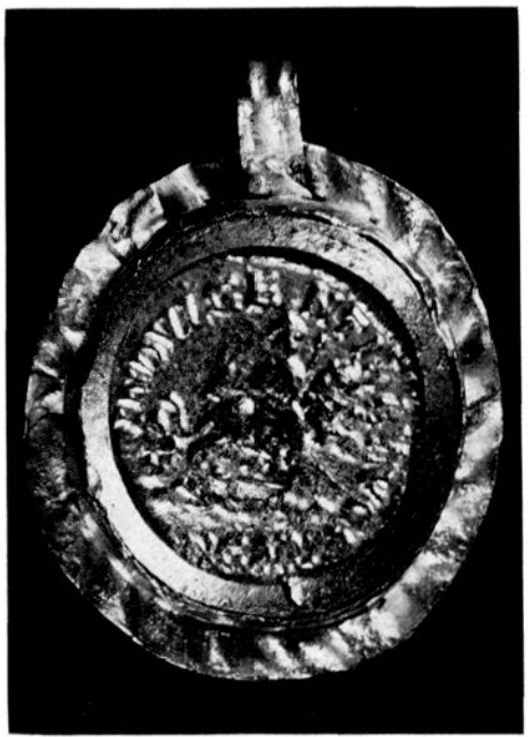

3 Mrabillon monelaire no 1 . Revers. (Ech. : :1).

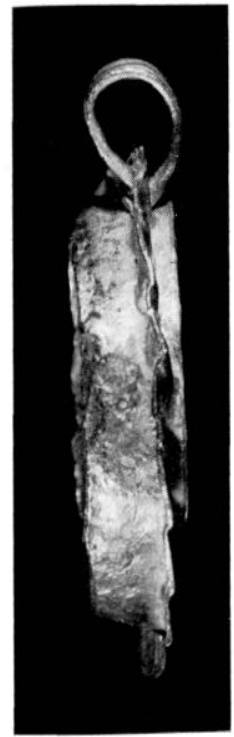

4 Mrdaillon monilaire no l. Chant. (Éch. ::1).

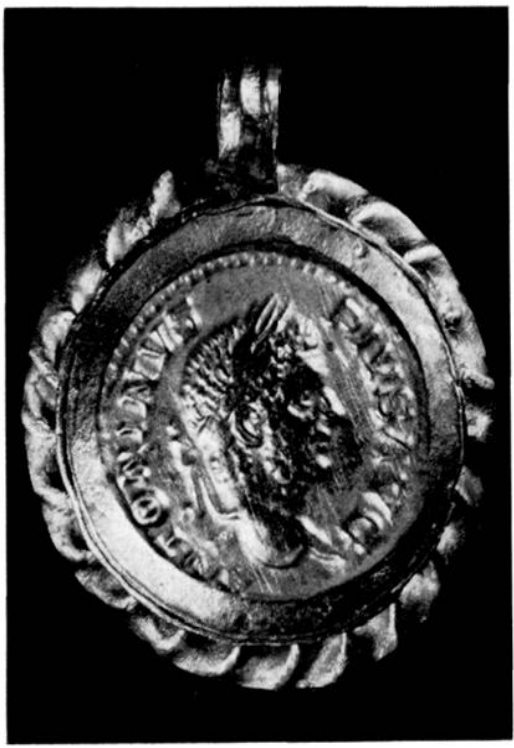

5 Vedaillon monétaire no 2. Arers. (Ech. : :1)

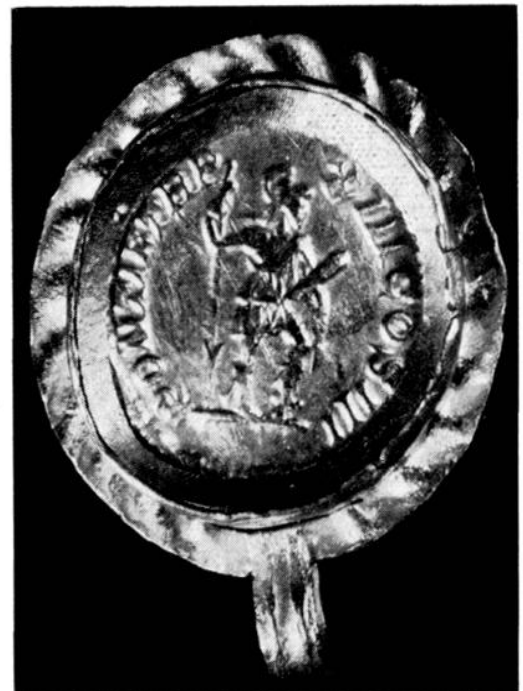

6 Védaillon monélaire no 2 . Revers. (Éch. 2:1).
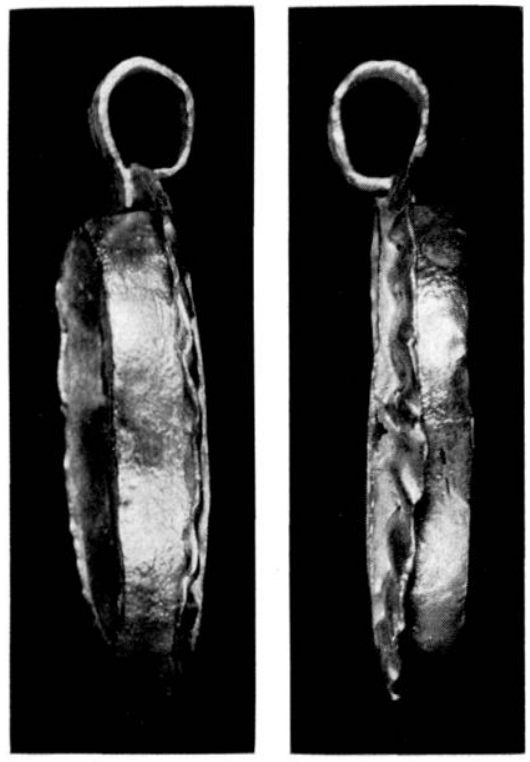

7 Médaillon monélaire $n^{0} 2$. Chant. (Ech. 2:1).
8 Védaillon monetaire no 2 . Chaml. (Ech. ::1). 


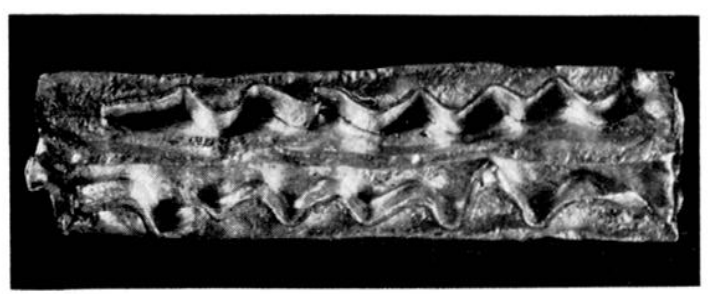

9 Coulant no 3. (Ech. : :1).

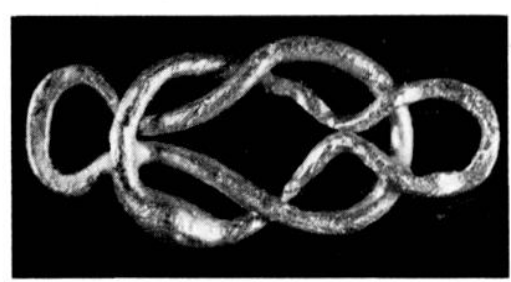

11 Noud d'llerakles $n^{0}$ 4. (Éch. 3:1).

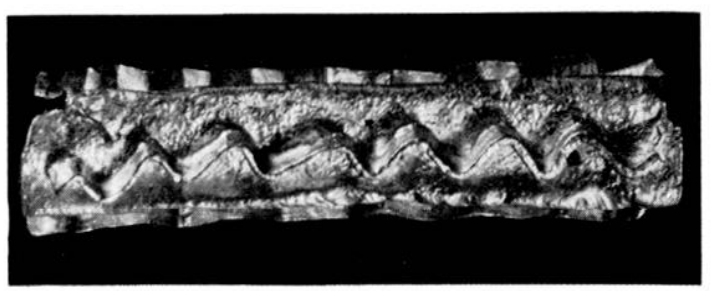

10 (Ooulant no 3. (Éch. 3:1).

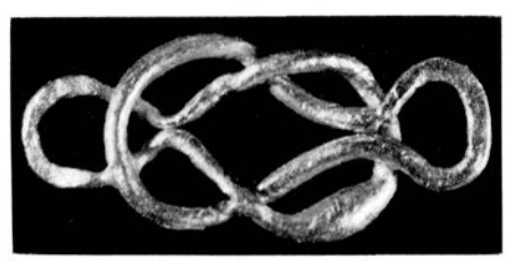

12 Naend d'Hérakles no 4 . (Éch. $3: 1$ ).

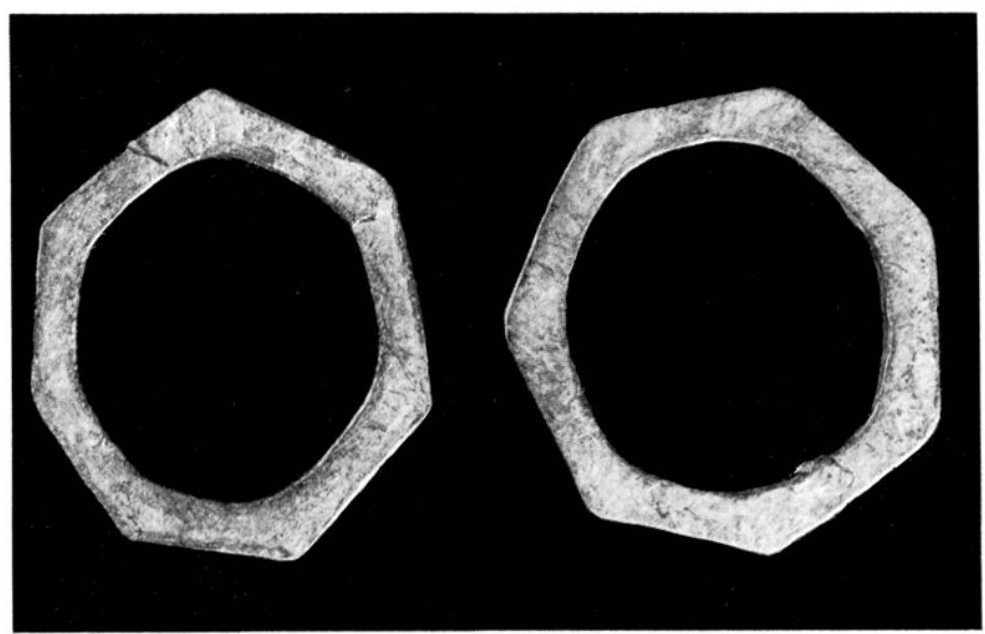

13 Heptagone no 5. (Éch. 3:1).

1.4 Heptagone n" 6. (E:ch. 3:1).

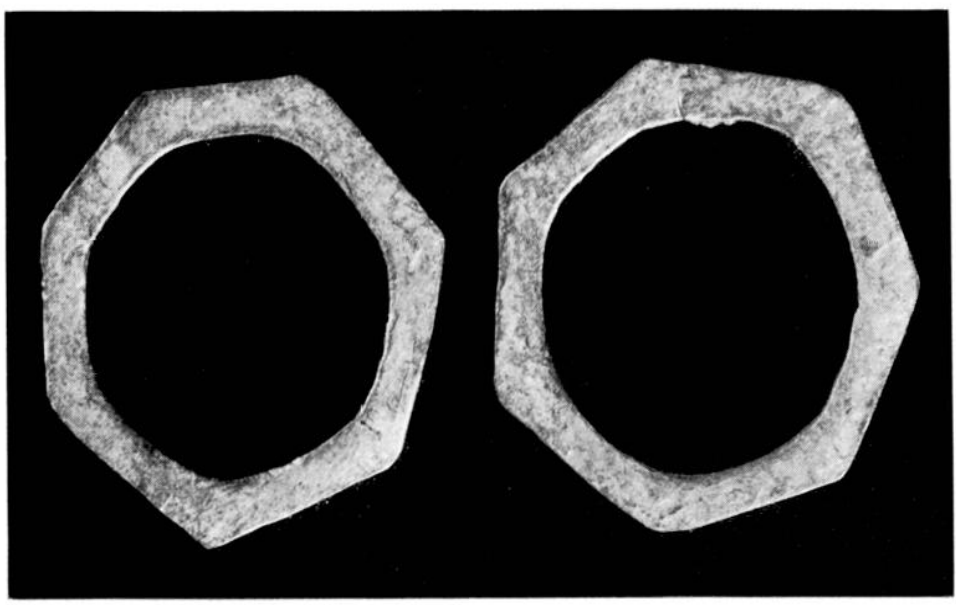

15 Heptagone no 5. (Éch. 3:1).
16 Heptagone no 6. (Éch. $3: 1)$.

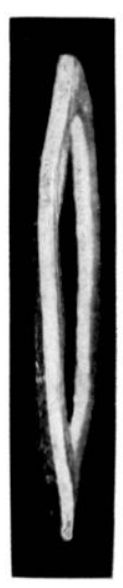

17 Heplagone $\mathrm{n}^{\circ} 5$

Chant. (Ech. $3: 1$ ). 
rainures de manière à façonner les cannelures irrégulières qui renforcent cet élément (fig. 20 et $21 ; i^{17}$. La découpe des bords a été faite au ciselet. L'ordre de montage de ces six éléments a probablement été le suivant : cylindre, soudure du biseau de l'avers, soudure de la collerette, soudure de la bélière, mise en place de la monnaie, sertissage du biseau du revers.

2. Médaillon monélaire (denier de Caracalla) (fig. 5 à 8 ot 19).

Dimensions : haut. totale : $30,5 \mathrm{~mm}$; diam. : 25) environ; épais. (cylindre) : 5 environ; poids : 7,52 gr. Atelier de Rome, année 210, type B.M.C. no 32.

D/ ANTONINVS PIVS AVG. Tête laurée à droite.

R/ PONTIF TR P XIII COS III. Virtus ou soldat debout, tête à droite.

Etat de conservation. Monnaie : longues rayures obliques sur les deux faces; coups; légère usure des saillants (fig. 19), la surface des dépressions présente quelques marbrures sur le revers. Cylindre : rayures; coups; trous dans la partie à droite de l'avers; très légère usure des bords par le port (fig. 8). Biseaux : rayures et coups. Collerette : déformation à droite du droit; surface grumeleuse. Pas d'usure par le port notable. Bélière : même observation.

Mélal. Monnaie : on fera les mêmes constatations que dans le cas précédent en ce qui concerne la différence de tencur en cuivre de la feuille de placage entre le droit et le revers. Tandis que les teneurs en argent sont presque identiques $(6,8$ et 6,5$)$, celles en cuire sont de 8,3 pour le droit et de 2,4 pour le revers. La différence de couleur est là encore perceptible. Les ors de la bélière et de la collerette sont assez semblables dans les deux pièces en ce qui concerne les taux d'argent et de cuivre :

\begin{tabular}{|c|c|c|c|c|c|c|c|c|c|}
\hline & $\mathrm{Ag}$ & $\mathrm{Cu}$ & Sn & $\mathrm{Pb}$ & $\mathrm{Zn}$ & $\mathrm{Gd}$ & $\mathrm{Fe}$ & $\mathrm{Ni}$ & $\mathrm{Mn}$ \\
\hline Bélière. & 7,3 & 4,3 & d & d & nd & $d$ & d & nd & nd \\
\hline Collerette.. & 6,4 & 2,9 & $d$ & $d$ & nd & nd & nd & $d$ & nd \\
\hline
\end{tabular}

On ne sait même pas si la teneur légèrement supérieure en cuivre de la bélière, plus résistante de ce fait que dans la pièce précédente, est le résultat d'un choix délibéré ou du simple hasard. On notera cependant qu'elle a mieux résisté à l'usure. En revanche, l'absence de zinc et de manganèse, les disparités en ce qui concerne le cadmium, le fer et le nickel prouvent que des ors de diverses provenances ont été utilisés pour ces bijoux (voir infra).

Technique de fabricalion. La technique de fabrication employée ici est dans ses grandes lignes la même que celle de la pièce précédente. On retiendra cependant des différences de détail.

Cylindre : la feuille est plus mince, plus ourlée du côté gauche de l'avers (fig. 7). Le

17 G. Nicotrsi, p. 112 (sur le "moulage à froid"), p. 155 (façonnage des demi-joncs). 
joint visible sur le rôté droit a été soudé à l'alliage. La surchauffe de la soudure a provoqué la fusion de la feuille à droite de l'avers, où l'alliage à souder a débordé largrement (fig. 8) ${ }^{18}$.

Collerette : la feuille presente des traces nettes de martelage sur une surface grumeleuse, sans travail de finition ultérieur. La soudure a souffert de la même surchauffe. Employée avec excès, rlle a débordé sur la partie droite du côté de l'avers, et s'est légèrement décollée ultérieurement (fig. 8 et 19).

Biseaux : la découpe est plus irréguliere. (On apercoit au bas de la figure 18 un débordement de sourlure caractéristique.

Bélière : plus épaisse (de 25) à 60/100e) et plus résistante à cause de son alliage (voir supra), elle est montée "en point d'interrogation" autour de la collerette qu'olle ne traverse pas.

3. Coulant (fig. 9, 10 et 22 ).

I)imensions : long. totale: : $23 \mathrm{~mm}$; larg. : 5) environ; poids : $1,11 \mathrm{gr}$.

Elal de conservalion. L'objet a probablement perdu ses renforts (voir ci-dessous p. 100, note 29). Less extrémités existantes sont déformées et déchirées par endroits. Ies còtés ont été enfoncés, peut-ètre par la pression de la terre, ce qui a porte en saillie les angles. On note de légères traces d'usure par le port sur les saillants, des rayures et des coups.

Mélal. Il est curieux de constater ici un taux d'argent particulièrement faible (de $1, \bar{y}$ à 1,9$)$ qui est sans doute le fait d'un or raffiné, qui a été allié à une notable proportion de cuivre, 3 pour le corps de la pièce el 4,9 pour le fil plat, plus résistant. llus intéressante est l'identité de composition, en ce qui concerne ces deux métaux, entre le corps de la pièce et la soudure bouillie $(\mathrm{Ag}=1,9,(\mathrm{iu}=3)$ qui prouve sans doute la perte de cuivre lors de la surchauffe dans la soudure. Sur l'extrémité de la pièce on relève l'analyse suivante:

\begin{tabular}{|c|c|c|c|c|c|c|c|c|c|}
\hline & $\lambda g$ & $\mathrm{Cu}$ & $\sin$ & $\mathrm{Pb}$ & $\mathrm{Zn}$ & $\mathrm{Cdl}$ & $\mathrm{Fe}$ & $\mathrm{Ni}$ & $\mathrm{In}_{\mathrm{n}}$ \\
\hline Coulant. & 1,9 & 3 & $d$ & $d$ & nd & nd & $d$ & nd & $d$ \\
\hline
\end{tabular}

dans laquelle on note l'absence parallèle du zine et du cadmium.

T'echnique de fabricalion. Le corps de l'objet est fait d'une feuille assez mince de 2 à $5 / 100 \mathrm{e}, 1 / 100^{\mathrm{e}}$ en certains endroits, assez peu adaptée à l'usage qui lui est demandé. Celle-ci a été pliée en quatre et soudée sur un angle (fig. 22). On a soudé de chant sur les quatre faces du parallélépipède un fil plat ondé de section variable découpé au ciselet dans une feuille de 1 à $3 / 10^{\mathrm{e}}$ d'épaisseur, la largeur variant de 4 à $7 / 10^{\mathrm{e}}$. La soudure en excès partout, a été surchauffée au point de bouillir et de déborder sur les quatro còtés (fig. 9). Sur l'un des côtés la surchauffe a provoqué des trous dans la feuille (fig. 10).

18 Ibid., p. 209, H. Hoffmaxi, P. Davidsox, Greel Gold, Boston 1968, p. 49. 


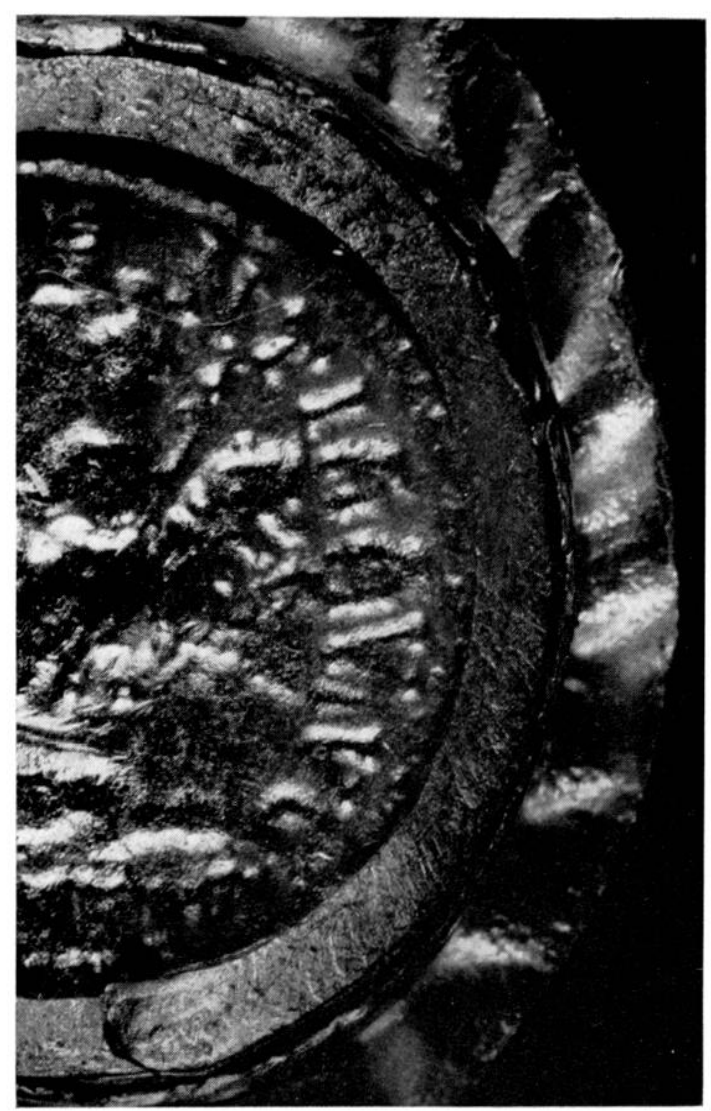

18 Medaillon monetaire no 1 . Tevers. Detail. (Éch. $;, 0: 5: 1)$.

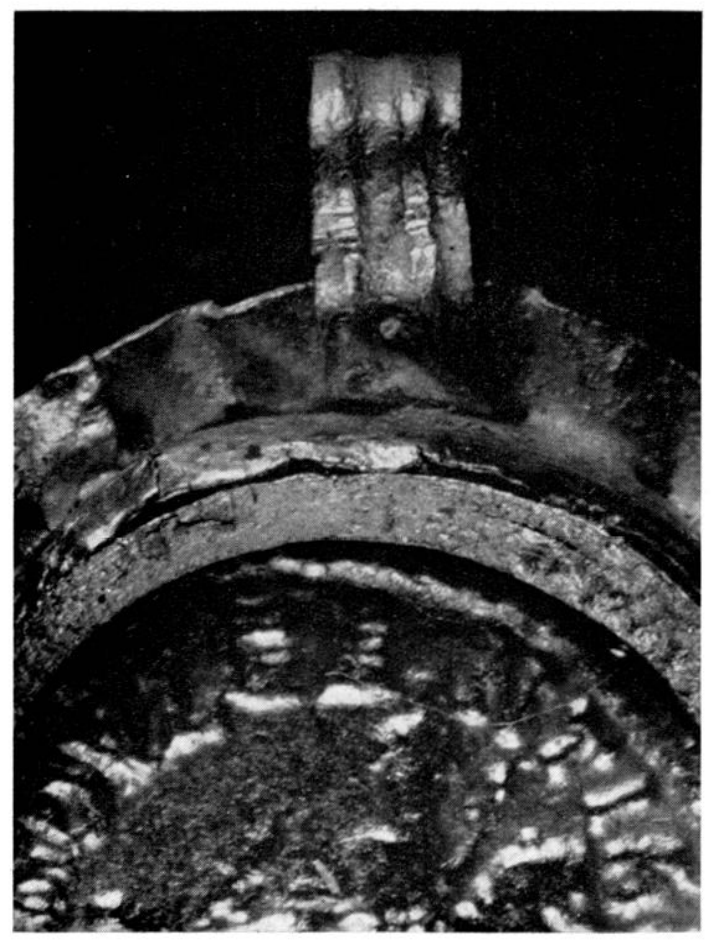

20 Medaillon monetaire $1^{\circ}$ I. Revers. Detail. (Eich. $, 0,2: 1)$,

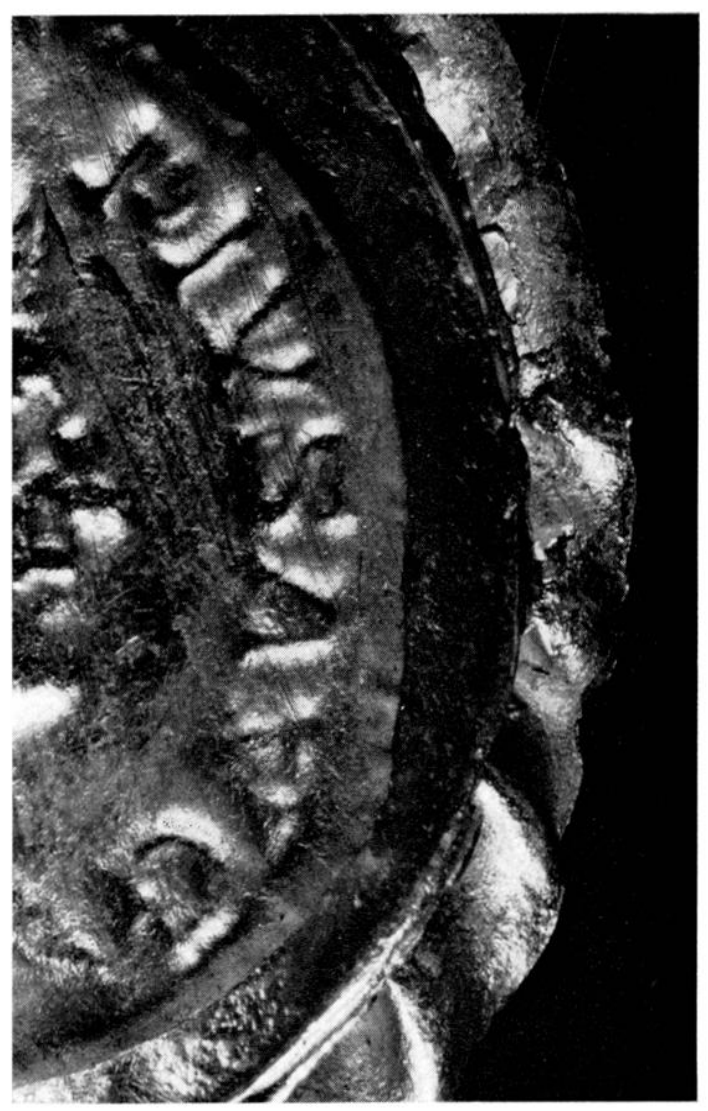

19 Védaillon monetaire no 2. Avers. Ditail. (Ech. $6,7: 1)$.

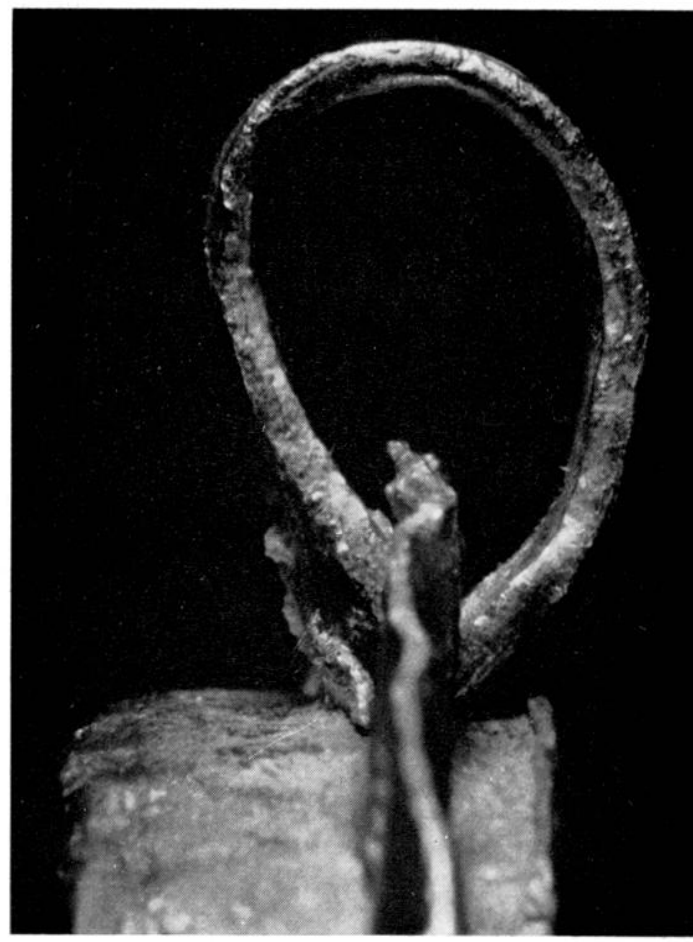

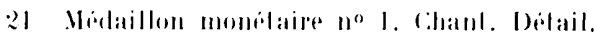
(E.ch. $8,7: 1)$. 
4. Noud d Iléraliles (fig. 11, 1: at : $: 3)$.

Dimensions : long. : 16.5) mm; larg. : (6.5) poids : 0,61 grr.

Elal de conservalion. Légere usure par le port des boucles des extrémites. Lsure des saillants.

Métal. On constate une diftérence notable entre les leneurs an cuive du corps de la

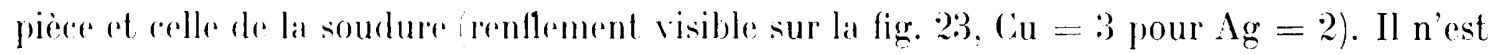
pas exclu malgré tout que celle-ci ait été appaurrie par une surrhauffe qui a dégradé le fil dans ses parties les plus fines. Voici l'analyse du corps :

\begin{tabular}{|c|c|c|c|c|c|c|c|c|c|}
\hline & $\Delta \underline{~}$ & $\mathrm{Cu}$ & Sn & $\mathrm{Pb}$ & $7 \mathrm{n}$ & $\mathrm{Crl}$ & $\mathrm{Fe}$ & $\mathrm{Xi}$ & In \\
\hline Norud d'Herakles. & 1 & 2,5 & d & $d$ & $d$ & d & $d$ & d & $d$ \\
\hline
\end{tabular}

qui comprend toute la série des traces que nous avons retenues. la faible teneur en argent dénote arec une quasi-certitude un or raffiné ${ }^{19}$.

T'echnique de fabrication. Cet objet est constitue de deux longueurs de fil mises en forme de double boucle. engagées l'une dans l'autre et soudées. Ce fil est sans conteste tordu et non tréfilé. cest-a-dire fabriqué à partir d'une bande d'épaisseur notable, tordue sur elle-mème20. La soudure a pu etre pratiquée en deux fois (soudure des boucles puis soudure de l'ensemble) ou en une seule fois avec une surchauffe qui a détéroré le fil.

5. Ileplagune (fig. 13, 15), 17 et :24).

Dimensions : diam. maximum : 15,4; épais. : :/10e anviron; poids : $0,64 \mathrm{gr}$.

Eklal de conservation. Rayures; un roup rérent (fig. 24); usure notable des angles et du bord interne par endroits, probablement par le port.

Mélal. I) Ho points d'analyse ont donne:

\begin{tabular}{|c|c|c|c|c|c|c|c|c|c|}
\hline & $\lambda \underline{r}$ & $\mathrm{Cu}$ & $\sin$ & $\mathrm{Pb}$ & $\mathrm{Zn}$ & $\mathrm{Crl}$ & $\mathrm{Fe}$ & $\mathrm{Ni}$ & In \\
\hline Premier heptagone.... & 8.9 & 1.6 & $d$ & $d$ & d & d & $d$ & nd & $d$ \\
\hline
\end{tabular}

Cette formule qui pourrait rorresponeler à un or naturel. se différencie neltement des préeredentes pareses leneurs an argent et en cuivere.

Technique de fabricalion. La pièce a elé decoupée au ciselet dans une feuille épaisse, puis elle a ité ouverte ef refermée, peut-ètre après un rétrécissement provoqué par le

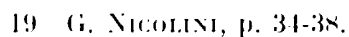

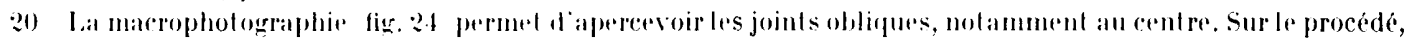

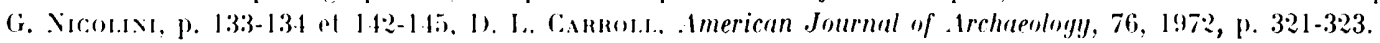




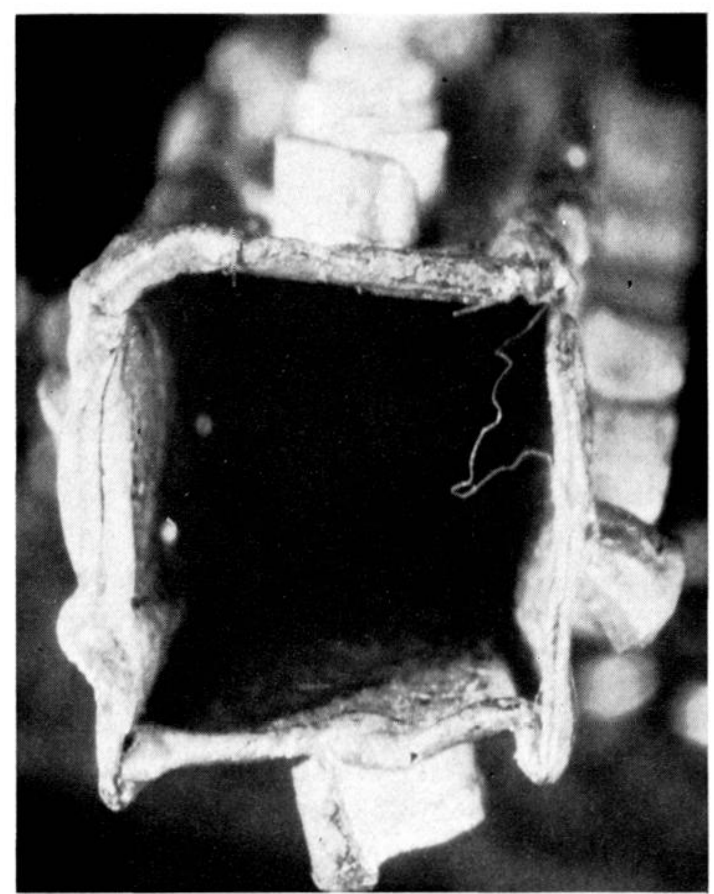

22 Coulant $n^{\circ} 3$, Extremite face. (Ech. 12:1).

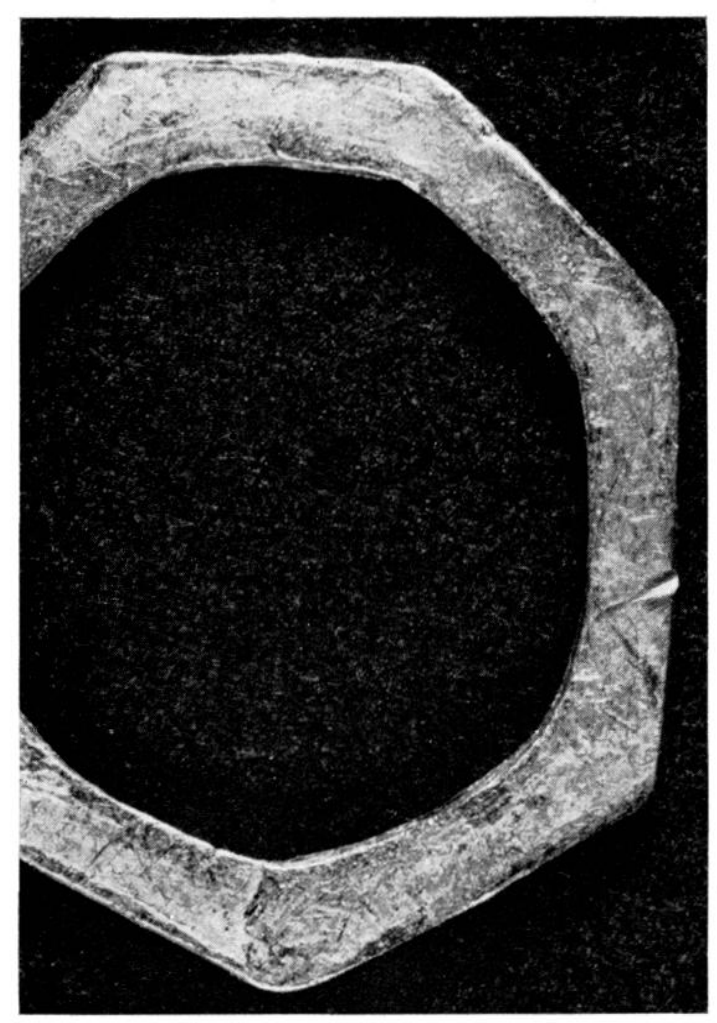

24 Heptarone $n^{\circ}$ 5. Detail. (Ech. $\left.;: 1\right)$.

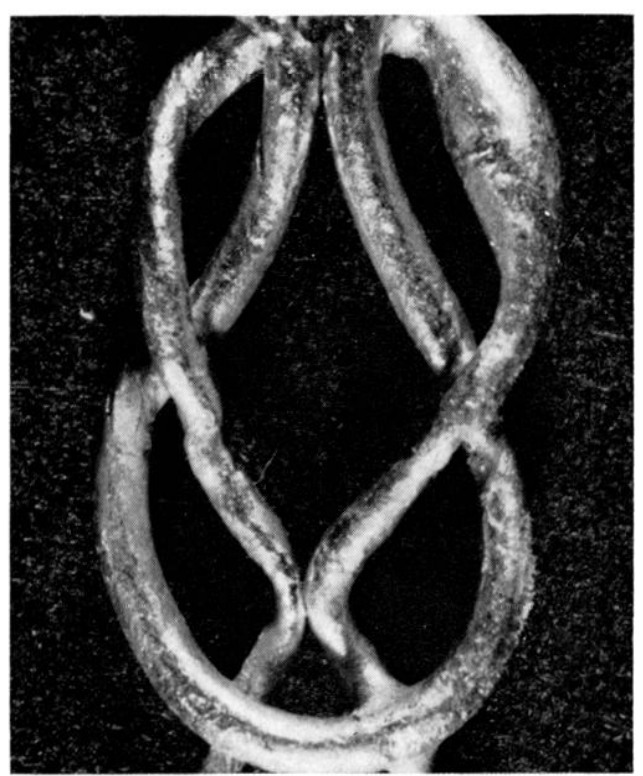

23 Toud d'Herakles no 4. Délail. (Ech. $7,3: 1)$.

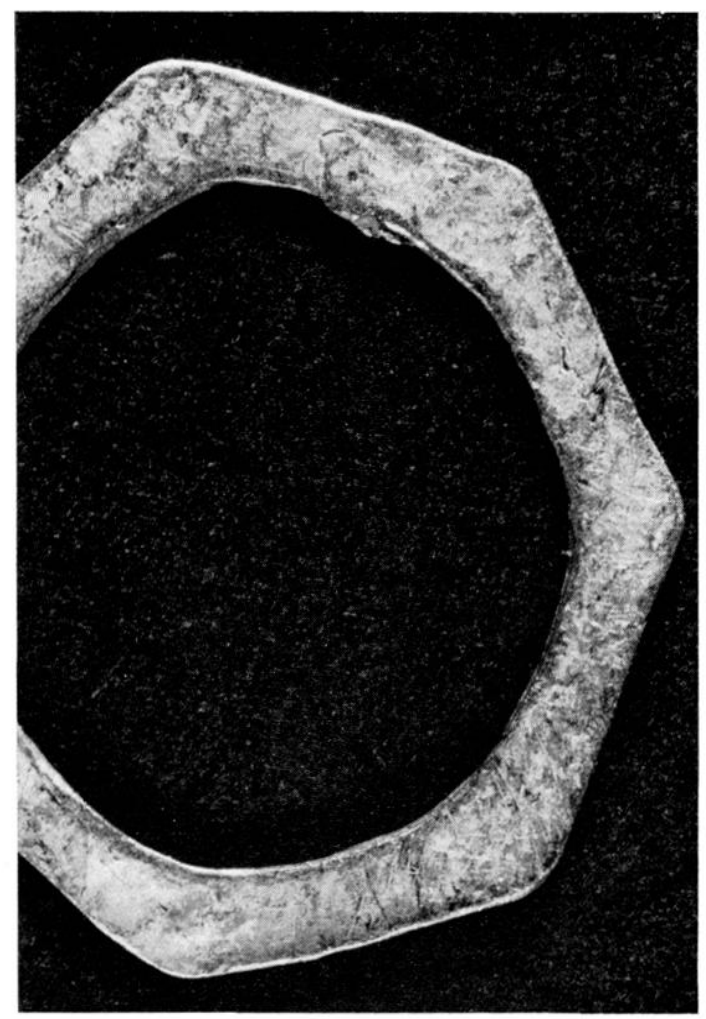

25) Heplagone no 6. Délail. (Ech. fi:1). 
recouvrement des extrémités de part et d'autre de l'ouverture. Le joint a été ensuite martelé et n'est probablement pas soudé, comme l'ont prouvé les analyses de la seconde pièce semblable (voir infra). Les barbes produites lors du découpage ont été ensuite martelées sur les deux côtés (fig. 24). Les raisons de cette ouverture nous échappent. On peut penser à l'hypothèse d'un rétrécissement pour obtenir les sept côtés à partir d'un polygone aux côtés plus nombreux. Plus vraisemblable est l'utilisation de ces pièces comme des maillons d'une chaine, re qui rendait obligatoire leur ouverture ${ }^{21}$.

\section{Second heplagone (fig. 14, 16 et 25$)$.}

Dimensions : diam. maxinum : $15,6 \mathrm{~mm}$; épais. : $5 / 10^{\mathrm{e}}$ environ; poids : $0,65 \mathrm{gr}$.

Élal de conservalion. Rayures et coups sur les deux faces; usure prononcée des angles par le port.

Mélal. Jeux points d'analyse ont été pratiqués, révélant une importante disparité du métal dans cette pièce. Le premier sur le joint, correspond à peu près aux teneurs de la pièce précédente, ce qui fait douter de l'hypothèse d'une soudure; le second, loin du joint, accuse une importante tencur en cuivre.

\begin{tabular}{|r|c|c|c|c|c|c|c|c|c|}
\hline & $\mathrm{Ag}$ & $\mathrm{Cu}$ & $\mathrm{Sn}$ & $\mathrm{Pb}$ & $\mathrm{Zn}$ & $\mathrm{Cd}$ & $\mathrm{Fe}$ & $\mathrm{Ni}$ & $\mathrm{Mn}$ \\
\hline 2e heptagone $1 \ldots \ldots \ldots$ & 8,5 & 1,8 & $\mathrm{~d}$ & $\mathrm{~d}$ & $\mathrm{~d}$ & $\mathrm{~d}$ & $\mathrm{~d}$ & $\mathrm{~d}$ & $\mathrm{~d}$ \\
$2 \ldots \ldots \ldots$ & 8,7 & 6 & & & & & & & \\
\hline
\end{tabular}

Cette disparité semble correspondre à un alliage mal mélangé lors de la fonte, comme le suggèrent d'ailleurs des marbrures à la surface du bijou.

Technique de fabricalion. Elle est identique à la précédente. L'intérêt de cette pièce par rapport à l'autre est dans la persistance des traces de martelage à la surface, visibles sur lia macrophotographic (fig. 25$)$ ).

Les bijoux de l'Houmeau permettent d'intéressantes observations sur les techniques de la bijouterie antique. En ce qui concerne le métal utilisé on remarquera d'abord des différences importantes dans les teneurs en argent et en cuivre, qui ne correspondent pas, dans l'ensemble, à une adaptation des formules naturelles ou s'il y en a, des alliages, à l'usage des piècess ou des différents éléments de celles-ci. On retiendra à titre d'exemple les teneurs de la pièce $n^{0} 1$ :

\begin{tabular}{|c|c|c|c|c|}
\hline & $\Delta \underline{\sigma}$ & $\mathrm{Cu}$ & & $\lg$ \\
\hline Ionnaie droit. & $6, \overline{5}$ & 4.3 & Biseau. . & 11 \\
\hline Monnaie revers. & 6 & 7,9 & Collerette.... & 6,8 \\
\hline Gylindre.............. & 6,4 & 4,7 & Bélière................... & 7,3 \\
\hline
\end{tabular}

21 Cous pensons aux chaînes à maillons plats découpés dans des feuilles, à peu près contemporaines, F. H. Marshalt, Calalogue of the jewellery in the depariments of antiquilies, British Museum, Londres 1911, no 2712, pl. LVI. 
Cette monture a probablement été exécutée à l'aide de fragments de diverses provenances dont disposait l'orfèvre, qui ont été travaillés et mis en forme pour l'occasion. La disparité entre les traces de métaux dans la collerette ct la bélière semble bien confirmer cette hypothèse (voir supra). L'orfèvre utilise done dans l'ensemble des ors assez purs de 843 à $888 / 1000$ e dont il ne tient compte ni de la résistance (bélière) ni de la couleur (biseau).

L'analyse des traces ouvre la voie à d'autres hypothèses. Considérons d'abord son aspect négatif. Il manque les métaux suivants : arsenic, bismuth, cobalt, titane, chrome, molybdène, tungstène, platine. mercure. lans l’etat actuel de la recherche, ces carences apparaissent encore peu significatives, elles sont néanmoins à retenir ${ }^{22}$. A contrario, on relève la présence générale de l'étain, que nous ne pouvons pas dosere ${ }^{23}$ et du plomb. Ce dernier métal, relativement rare, pourrait être un indice révélateur d'une provenance géographique relativement circonscrite de tous les ors utilisés dans le trésor. Le zinc, très rare dans les ors antiques, est associé au cadmium, sauf dans la bélière du second médaillon, où l'on constate la présence du cadmium ct l'absence de zinc. Ils manquent tous deux dans la collerette de celui-ci, el dans le coulant. La présence de cadmium peut paraître surprenante, car il n'a pas été détecté jusqu'à aujourd'hui dans les ors antiques ${ }^{24}$. Nous pensons qu'elle devrait être recherchée systématiquement dans les analyses. L'association éventuelle de ce métal avec le zinc pourrait ètre étudiée également avec profit. Pour l'instant, toute hypothèse sur la présence du cadmium dans les ors antiques nous semble prématurée ${ }^{25}$.

Enfin, il resterait a expliquer le travail et la technique de ces bijoux. Nous avons vu combien le choix des ors était parfois peu judicieux. Le travail de la feuille --- martelage et découpage - est très sommaire particulièrement en ce qui concerne ces pièces 1 à 3. Les épaisseurs sont trop faibles (cylindres, coulant). Les soudures sont excessives et mala-

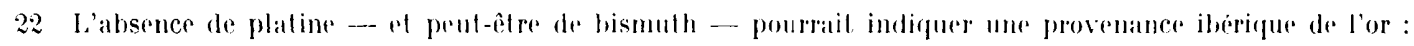
A. Hantuaxi, P. Kals, Archivo de Prehistoria Lelantina 12-1969, p. 91-95. L'absence de mercure peut signifier que l'or n'a pas dete oblenu par amalgame.

23 Les groupes d'or se definissent d'abord par la teneur en etain, G. Nicorivi, p. 31-35. Nous n'avons pas fait figurer dans les tableaux que nous publions, le sodium, le magnisium, l'aluminum et le silicium, qui sont presents sous forme de traces dans toutes les analyses pratiques sur ce trosor par le Jaboratoire des . Wusees de France.

24 I.e cadmium se trouve associe ain zinc dans les soudures modermes. Ies deux metaux abaissent la temperature de fusion de celles-ci.

25. Les seules que l'on puisse avancer sont d'ordre mineralogique. En premire lieu, on sait que l'or peut être present dans les gisements de zinc blende, associé géneralement au cadmium nous remercions Pierre Nicolini de nous avoir fourni ce renseignement . La blende a ete exploité à l'epoque romaine, ou le zinc entrait dans la composition de l'aurichalque (- laiton. Le mélange se faisait probablement non par alliage des métaux élaborés mais par cémentation des minerais conversation avec le Pr. I. Vandevivere, Louvain, mars 1981, Lors de l'exploitation de la blende, ou de sa transformation, l'or apparassant sous forme de sous-produit devait contenir de notables traces de zinc ou de cadmium. Mais la blende pouvait aussi bien être pexploité pour sa teneur en or. Ainsi un ichantillon riche en blende préleve sur les exploitations antiques de Zarehshuran Iran occidental, a quelques kilometres du lieu de trouvaille du trésor de Ziwiyé avait une leneur de $20 \mathrm{~g}$ à la tonme P. Baraxis, (í. P'issigr, Origine de l'or de Zarehshuran, dans Bull. Soc. Française de Miniralogie el Cristallographie 95-1972, p. 697. Il est fort probable (fue cet or contenait des traces de zinc et de cadmium au moment de son extraction. Enfin on peut se demander pourquoi le cadmium a ete si peu détecté dans les ors antiques travilles. Outre qu'il n’a pas itr systemaliquement recherché comme nous l'avons dit, cette carence a sans doute une rason physiqur : dans la coupellation de l'or, ou son affinage, le zinc et le cadmium, entre autres métaux, disparaissent le plus sowvent à cause de leur ligrerete cet fusibilite. Il serait d'ailleurs interessant de savoir dans quelle mesure les echantillons d'or antique à cadmium ont ete fondus ou travailes à basse temperature. Il est certain qu'un or nalurel non fondu a plus de chances d'avoir conservi des lraces de ces metaux volatiles. Nous remercions vivement MII. P. Bariand ed J. Goffroy de nons avoir apporte ces precisions. 
droitement surchauffées dans les quatre premieres pièces. La parenté de technique entre celles-ci semble indiquer une identite d'atelier. voire de main, particulièrement dans le montage (le battage dess feuilles du second médaillon est plutôt moins maladroit). Les heptagones pourraient avoir ete travaillés dans un atelier différent, comme le suggèrent le martelage de la feuille of l'assemblage sans soudure.

En tout etat de cause il nous semble qüil sagit de la production d'officines locales, imitant les réalisations de qualite qui avaient rours à l'epoque, comme nous allons le constater.

G. X.

\section{Essai donterpritatiox.}

Le trésor de l'Houmeau comporte au moins quatre éléments que l'on peut aisément rattacher à une catégorie de bijoux bien eonnus à l’époque romaine. Ce sont les deux pendentifs monélaires, le coulant et le motif en noud d'Héracles. Les deux pendentifs qui se composent d'une monnaie entourée d'une monture en or avec bélière de suspension faisaient vraisemblablement partie d'un de ces colliers monétaires dont il existe de nombreux exemples dans l'orfèverie romaine, au $\mathrm{II}^{\mathrm{e}}$ siècle en particulier. Les deux médaillons récemment découverts à l'Iloumeau, présentent cependant une particularité remarquable : les monnaies ne sont pas des aurei comme dans la très grande majorité des pendentifs monétaires mais des deniers d'argent recouverts d'une feuille d'or selon un procédé décrit plus haut (roir (i-dessus, p. 89)). Il ne semble pas que l'on ait, sauf peut-être pour des époques plus tardives, beauroup d'exemples d'un tel procédé. Les bijoux monétaires, les bagues en particulier. peuvent parfois être composés d'une monnaie d'or et d'une monture en argent ou l'inverse mais l'argent est alors bien visible et non caché par une feuille d'or. Cependant un bijou trouvé à Autun ast vraisemblablement également constitué d'un denier de Maximin rerouvert dune feuille d'or et entouré d'une monture ${ }^{26}$. Lne étude attentive de cet objet permettrait sans doute de montrer si la façon de fixer la feuille d'or est la mème que pour les deux monnaies de l'Houmealle.

La monture dans laquelle s'inserent les deux deniers plaques de l'Houmeau est du type habituel aux pendentifs monetaires : un cylindre et deux biseaux d'or maintiennent la monnaie, une collerette ourragée est sourlée au cylindre. Les deux collerettes identiques, sont simplement constituées d'une etroite bande d'or façonnée de manière à lui donner l'aspect d'un ruban gaufré. La beliere à cannelures moulurées est soudée sur la collerette.

Cette collerette-ruban apparait comme l'un des types les plus simples que les orfèvres romains aient employés pour la monture des pendentifs monétaires. Ceux-ci présentent en effet plus souvent dess rolleretles beaucoup plus ouragées à décor découpé en opus

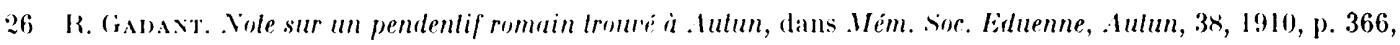

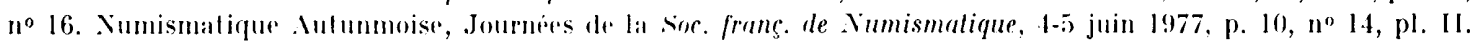


inlerrasile comme celles qui entourent, parmi beaucoup d'autres, les médaillons du collier de Naix $^{27}$ ou ceux découverts à Beaurains ${ }^{28}$.

Le troisième élément qui doit aroir fait partie d'un collier monétaire est le petit tube creux, de section carrée, orné sur chaque face d'un fil plat formant un décor de zig zag. Cé tube constitue sans aucun doute un coulant de séparation qui, enfilé sur une chaîne, permet de maintenir espacés les médaillons monétaires. (On trouve en effet fréquemment ce type de tube associé à des pendentifs monétaires. les colliers de Naix et de Beaurains en fournissent des exemples ${ }^{29}$. L'objet trouvé à l'Houmeau est probablement incomplet, il semble en effet que ces coulants soient, le plus souvent, munis de renforts moulurés ou lisses, soudés aux extrémités.

Le dernier objet qui peut avoir fait partie du collier est le nœud d'Héraclès. Il s'agirait alors de l'élément de fermeture du collier. La chaîne à laquelle étaient suspendus les pendentifs aurait comporté à une extrémité le nœud et à l'autre un crochet. Il est très rare que les colliers monétaires aient été conservés intégralement de sorte que nous connaissons mal les modes de fermeture de ces bijoux. Il existe cependant au moins un autre type de fermoir, composé d'un médaillon à deux bélières latérales, ce qui semble être le cas pour un collier, complet, conservé à Kansas City ${ }^{30}$ ou pour les colliers que l'on peut reconstituer comme celui de Beaurains ${ }^{31}$.

On connaît d'autre part plusicurs exemples de colliers d'époque romaine qui sont formés d'éléments en forme de nœuds d'lféraclès alternant avec des pierres de couleur, des prismes en racine d'émeraude ${ }^{32}$ ou des saphirs ${ }^{33}$. I)ans ce (as, le fil constituant le motif est plat et non circulaire comme pour le bijou de l'lloumeau.

Les deux derniers objets du trésor de l'Houmeau, les deux heptagones, sont, à l'inverse des quatre premiers, des éléments beaucoup plus étranges ct que l'on ne peut ranger dans des séries connues. Ils sont de trop petites dimensions pour que l'on puisse penser à des éléments de monture de monnaies, ainsi que l'on serait tenté de le faire au premier abord et l'on ne voit pas bien leur rapport avec les éléments du collier. On peut envisager l'hypothèse de l'utilisation de ces deux éléments comme maillons d'une chaìne bien que l'on ait peu d'exemples de tels maillons. On peut aussi rapprocher ces heptagones de trois petits bijoux conserves au Vuse historique de la ville de Budapest. Ce sont trois octogones assez irréguliers, apparemment découpés dans une feuille d'or. de dimensions assez voisines de nos heptagones. Ils proviennent du site romain d'Aquincum et ont été trouvés dans une tombe datée du $v^{\mathrm{e}}$ s. ${ }^{34}$. La destination de ces bijoux n'est pas connue. (On pourrait penser à des éléments cousus sur un voile ou un vètement.

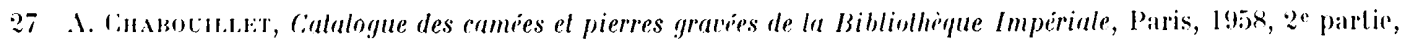

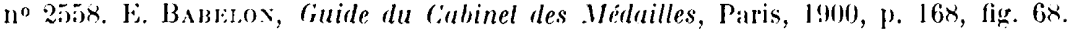

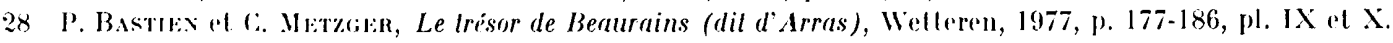

29 P. Bastu:x ol C. Ml:tzGise, op. cil., p. 177, pl. IX, 23.

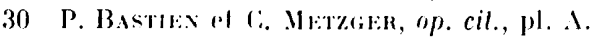

31 P. Bastusx of c. Merzgien, op. cil., p. 179, pl. IX, 3.

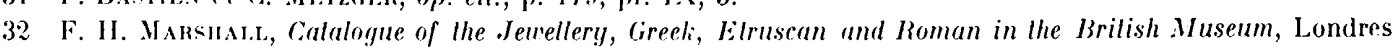
$1911,1^{0} 2730$, pl. IX.

33 A. Grempinatik, Schmuclatbeilen in Edelmetall, 11, Berlin, pl. 31, 4.

34 Renseignements aimablement fournis par .I me Klara Poczy, Directrice des fouilles d'Aquincum. 
Les bijoux de l'Houmeau apparaissent donc comme quelques éléments d'un trésor plus important. Le collier monétaire est de toute façon incomplet puisque la chaîne manque - il est vraisemblable qu'il existait plusieurs pendentifs monétaires encore que l'on ne puisse donner un nombre moyen pour les pendentifs composant de tels colliers. Ce sont en outre des objets d'artisanat assez commun, cherchant peut-être à imiter des exemplaires plus luxueux dans la matière et plus raffinés dans le travail d'orfèvrerie. L'ensemble de ces bijoux ainsi que le contexte de la trouvaille (cf. ci-dessus, p. 86) peut indiquer une date du milieu ou du début de la deuxième moitié du in ${ }^{\mathrm{e}}$ siècle.

C. M.

\section{J. Flouret, G. Nicolixi et C. Metzger.}

\section{AI)I:NDUM}

Une seconde série d'analyses pratiquées par le Laboratoire des Ilusées de France, que nous remercions une fois de plus des nombreuses indications qu'il a bien voulu nous fournir, a donné des résultats voisins des premiers sur des points d'étincelage différents. Toutefois, ceux-ci contredisent. les observations que nous avions formulées sur l'absence de certains corps (voir supra, p. 98, note 22). On a décelé la présence d'arsenic dans la bélière des deux médaillons, de titane dans la collerette du premier $\left(n^{0} 1\right)$ el dans le premier heptagone $\left(n^{0} 5\right)$, de chrome dans la collerette du $n^{0} 1$, de platine dans la bélière des $n^{0 s} 1$ et 2 , le cylindre du $n^{0} 1$ el le noud d'Iléracles $\left(n^{0} 4\right)$. Enfin, le mercure apparaît dans les deux bélières et dans la collerette du second médaillon ( $\left.\mathrm{n}^{0} 2\right)$. Ainsi ces dernieres analyses non seulement confirment l'existence de plusieurs qualités d'or selon les diverses pièces ou parties de pièce. mais aussi des différences perceptibles dans les traces suivant les points choisis a l'intérieur mème de celles-ci, distants quelquefois de seulement quelques millimètres. A la vue de ces résultats. nous devons donc insister sur la nécessité de pratiquer de multiples analyses sur un même bijou. particulièrement dans le cas où il est composé de plusieurs éléments, pour avoir une idée relativement juste de sal composition. Ine analyse unique ne permet de déceler autre chose que la présence de certains corps à un endroit donné. Yous formulons le souhait que de nombreuses analyses non destructives a raison de plusieurs points par bijou soient faites et publiées.

G. X. 